\title{
On the effect of local sample slope during modulus measurements by contact-resonance atomic force microscopy
}

\author{
K. Heinze, ${ }^{*, \mathrm{a}}$, O. Arnould ${ }^{\mathrm{c}}$, J.-Y. Delenne ${ }^{\mathrm{b}}$, V. Lullien-Pellerin ${ }^{\mathrm{b}}$, M. Ramonda ${ }^{\mathrm{d}}$, M. George, \\ ${ }^{a}$ L2C, University of Montpellier, CNRS, Montpellier F-34000, France \\ b IATE, University of Montpellier, INRA, CIRAD, Montpellier SupAgro, Montpellier, France \\ ${ }^{\mathrm{c}}$ LMGC, University of Montpellier, CNRS, Montpellier, France \\ ${ }^{\mathrm{d}}$ CTM-LMCP, University of Montpellier, Montpellier, France
}

\section{A B S T R A C T}

Contact-resonance atomic force microscopy (CR-AFM) is of great interest and very valuable for a deeper un-derstanding of the mechanics of biological materials with moduli of at least a few GPa. However, sample surfaces can present a high topography range with significant slopes, where the local angle can be as large as $\pm 50^{\circ}$. The non-trivial correlation between surface slope and CR-frequency hinders a straight-forward interpretation of CR-AFM indentation modulus measurements on such samples. We aim to demonstrate the significant influence of the surface slope on the CR-frequency that is caused by the local angle between sample surface and the AFM cantilever and present a practical method to correct the measurements. Based on existing analytical models of the effect of the AFM set-up's intrinsic cantilever tilt on CR-frequencies, we compute the non-linear variation of the first two (eigen)modes CR-frequency for a large range of surface angles. The computations are confirmed by CR-AFM experiments performed on a curved surface. Finally, the model is applied to directly correct contact modulus measurements on a durum wheat starch granule as an exemplary sample.

Keywords:

AFM

Contact resonance

Indentation modulus

Surface slope

Nano-mechanics

\section{Introduction}

The mapping of the mechanical properties of micrometre- and submicrometre-sized structures has become available in the recent years by the technological advancement of a number of methods based on atomic force microscopy (AFM). Due to the unlimited scope of applications, such techniques have already been applied in very different areas, such as cell wall stiffness in plants [1-3] or material science research on composites from natural polymers [4-6]. Wheat grains are another natural composite material, for which the knowledge of the micro-scale elastic properties of its components would greatly benefit the understanding and accurate simulation of the grains' milling behaviour [7].

One of the AFM-based nano-mechanical characterization methods is contact-resonance atomic force microscopy (CR-AFM). The elastic and viscous properties of the sample are inferred from the modal response of the AFM cantilever, which is in contact with the surface through the AFM tip, while the sample is subjected to a sinusoidal ultrasonic vibration of low amplitude [8,9]. This technique has especially been of great interest since it was shown that vibrational mode frequencies were very sensitive to changes in the indentation modulus of the sample
[10]. Despite the great potential of the technique, one has to be aware of the limitations before relevant information on the sample's mechanical properties can be extracted. The difficulties linked to the topography of the surface and the badly known geometry of the contact area are probably the most substantial and the most hindering, especially since the delicacy and the heterogeneous nature of the samples can limit the preparation of truly flat surfaces. Mazeran et al. [11] pointed out, for phase interpretation in tapping mode, how cautious one should be if the mechanical measurements are correlated with the sample's topography. In CR-AFM in particular, the resonance frequencies of the cantilever-tip-sample system are highly dependent on its exact geometry. Stan and Cook [12] studied for instance, how roughness can be taken into account for a granular material at the nanometric scale based on accurate Scanning Tunnelling Microscopy acquisitions of the sample topography and a model which takes into account multiple asperity contacts. Such a level of resolution is necessary when one hopes to get quantitative determination of the mechanical properties at the nanometric scale, but is rarely possible to reach. For most samples, the amplitude and wavelength of the roughness over a micron-sized area are fortunately smaller than the local radius of the AFM tip, so that such precise measurements are not

\footnotetext{
* Corresponding authors.

E-mail addresses: karsta-marlies.heinze@umontpellier.fr (K. Heinze), matthieu.george@umontpellier.fr (M. George).
} 
necessarily needed. However, the samples often present long range variations of the local slope, which constitute an important and easily measurable parameter of the contact geometry that should be addressed to improve the mechanical measurements. Passeri et al. [13] demonstrated the significant influence of the angle between the cantilever-tip and the surface on the frequency modes in CR-AFM. Yet, the only angle, which is usually taken into account, is the one directly resulting from the tilt of the cantilever in AFM devices. A possible slope of the surface is generally neglected, although it can induce local changes of the tipsurface angle, which can easily reach several tens of degrees. The nontrivial correlation between surface slope and CR-frequency then hinders a straight-forward interpretation of CR-AFM indentation modulus measurements.

In this paper we study the influence of the local surface slope on nano-mechanical measurements by CR-AFM. We propose a practical method to correct the measure by taking into account the topography, which is acquired simultaneously to the CR-frequencies. This correction method is based on a simplified model of the vibrating cantilever to emphasize the effect of the local slope. It can easily be implemented using more advanced models of the vibrating cantilever and should also be easily adaptable to other experimental techniques, i.e., acoustic and ultrasonic AFM [14-19], PeakForce ${ }^{\mathrm{TM}} \mathrm{QNM}^{\mathrm{TM}}$ [20] or $\mathrm{QI}^{\mathrm{TM}}$ [21], force modulation (FMM) [22] or any other force volume (FV) and force distance (FD) like modes, which are very likely exposed to the same problem.

The correction method was successfully applied to mechanical measurements on a biological surface, whose high slopes so far inhibited the correct interpretation of CR measurements: a cut starch granule within the sectioned endosperm of a wheat grain.

\section{Theory}

\subsection{Analytic determination of the vibration eigenmodes}

The key principle of CR-AFM is that the resonance frequencies of the cantilever are strongly dependent on the stiffness of the contact between the AFM tip and the surface. This contact stiffness is linked to the mechanical properties of the sample surface and to the contact area, which is mainly influenced by surface topography, tip shape, applied force and adhesion force. Frequency shifts can therefore reveal even small changes in the mechanical properties of the scanned surface, provided that a good compromise between stability of the tip-surface contact, sensitivity of the frequency measurement and sensitivity of the frequency shift response to contact stiffness changes are reached. The vibrational response of the cantilever can be modelled with the elastic equation of motion of a rectangular beam in the frame of the EulerBernoulli theory, with boundary conditions depending on the geometry of the cantilever-tip-surface system. It is a well-studied problem, resulting in a series of resonances corresponding to the different vibrational eigenmodes of the cantilever. Numerical solutions have been developed to obtain the amplitude and phase of the cantilever's vibrational response as a function of the angle and considering the entire complex geometry of the tip-cantilever system during contact-resonance measurements. Such in-depth theoretical analysis of the system can be found in the literature $[8-10,12,13]$.

Due to the numerous parameters that have a strong non-linear influence on the vibrational response of the cantilever, the application of these works to correctly analyse CR-AFM measurements is not straightforward. The present study focuses on the influence of the local slope of the surface on the measured CR-frequencies. We thus take benefits from the above mentioned global studies to provide a way to practically obtain quantitative measures on samples with surface angles as high as $50^{\circ}$. The overall geometry of the cantilever-tip system in contact with a surface is schematically presented in Fig. 1, along with a simplified version, which focuses on the effect of local slope angle. We will demonstrate hereafter that these simplifications allow to soundly fit the

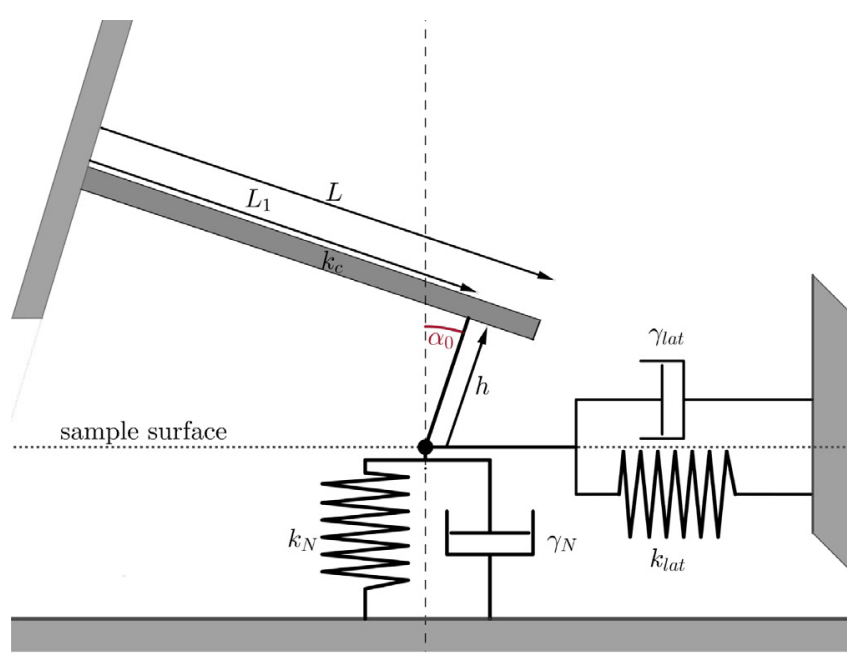

(a)

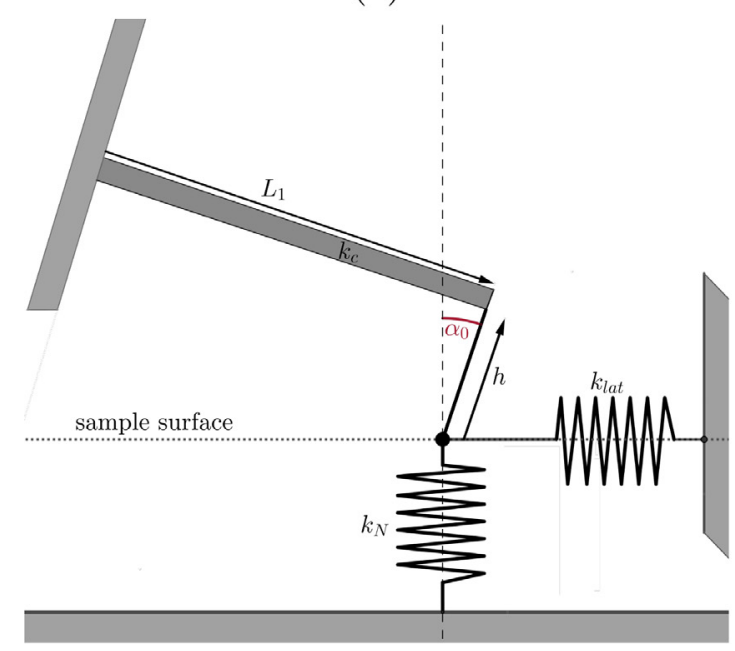

(b)

Fig. 1. Simplified elastic beam model of the cantilever and AFM tip in contact with a sample surface. (a) The full system is characterized by the cantilever length $L$ and stiffness $k_{c}$, the AFM tip height $h$, the surface's normal stiffness $k_{N}$ and lateral stiffness $k_{\text {lat }}$, the normal lateral damping, $\gamma_{N}$ and $\gamma_{\text {lat }}$ and the angle $\alpha$ between tip and surface. (b) The simplified model does not take into account damping, lateral forces, and the cantilever part extending the centre of the tip.

experimental results. Damping of the cantilever is not taken into account in the model, as we focus only on the determination of the resonance frequencies of the different vibration modes. They are sufficient for the determination of the indentation modulus, and the effect of damping of the cantilever on the resonance frequencies is assumed to be negligible. Similarly, damping $\gamma$ (cf. Fig. 1a) of the material of the sample is assumed to have a negligible effect on the measured frequencies and is also not taken into account. Rabe [8] have shown that the position of the tip along the cantilever length strongly influences the vibrational frequencies during contact and that the correct analytical model should involve both the total length $L$ of the cantilever and the real distance $L_{1}$ between the base of the cantilever and the exact tip location (cf. Fig. 1a). A sound simplification can be made, taking into account only the base-tip distance $L_{1}$ in the calculation assuming that the cantilever extension after the tip has a negligible effect on the resonance frequencies (cf Fig. 1b). One of the main difficulties in the analysis is the description of the normal and lateral forces that constitute the contact between the tip and the surface. While the normal stiffness $k_{N}$ of the tip-surface contact is always present and can be 
usually described with the well-documented models available in contact theory, the lateral stiffness $k_{\text {lat }}$ depends on the type of contact (free sliding, friction, pinning) that occurs between the tip and the surface, and is difficult to anticipate. A general way to address the lateral forces is to express the lateral stiffness as proportional to the normal one, $k_{\text {lat }}=\lambda k_{N}$, and to confront the model's results with increasing values of the coefficient factor $\lambda$, from 0 (free-sliding contact) to more than 1 (pinning) [8], to actual experimental results.

Describing the vibrations with an elastic beam model and the specific boundary conditions, one obtains a system of equations, whose non-trivial solutions are obtained independently of the excitation amplitude when the system's determinant is equal to 0 . This leads to solve the characteristic equation [8]:

$$
\begin{aligned}
& \left(\beta L_{1}\right)^{4}\left[1+\cos \left(\beta L_{1}\right) \cosh \left(\beta L_{1}\right)\right] \\
& +\left(\beta L_{1}\right)^{3} C_{1}\left[\sin \left(\beta L_{1}\right) \cosh \left(\beta L_{1}\right)+\cos \left(\beta L_{1}\right) \sinh \left(\beta L_{1}\right)\right] \\
& +2\left(\beta L_{1}\right)^{2} C_{2} \sin \left(\beta L_{1}\right) \sinh \left(\beta L_{1}\right) \\
& +\left(\beta L_{1}\right) C_{3}\left[\sin \left(\beta L_{1}\right) \cosh \left(\beta L_{1}\right)-\cos \left(\beta L_{1}\right) \sinh \left(\beta L_{1}\right)\right] \\
& +\left(C_{1} C_{3}-C_{2}^{2}\right)\left[1-\cos \left(\beta L_{1}\right) \cosh \left(\beta L_{1}\right)\right]=0,
\end{aligned}
$$

$$
\begin{aligned}
\text { with } C_{1} & =\left(\frac{h}{L_{1}}\right)^{2} \frac{3 k_{N}}{k_{c}}\left(\sin ^{2} \alpha+\lambda \cos ^{2} \alpha\right) \\
C_{2} & =\frac{h}{L_{1}} \frac{3 k_{N}}{k_{c}} \sin \alpha \cos \alpha(\lambda-1) \\
C_{3} & =\frac{3 k_{N}}{k_{c}}\left(\cos ^{2} \alpha+\lambda \sin ^{2} \alpha\right) .
\end{aligned}
$$

The solutions $\beta_{n}$ of this equation can be obtained numerically as a function of the geometrical parameters cantilever length $L_{1}$, tip height $h$, angle $\alpha$ between tip and surface, of the ratio $\lambda$ between normal and lateral stiffness, and of the ratio $k_{N} / k_{c}$ between normal contact stiffness $k_{N}$ and cantilever stiffness $k_{c}$. The solutions are the wavenumbers $\beta_{n}$ of the spatial vibration modes $\left(n \in \mathbb{N}^{*}\right)$. Wavenumber $\beta_{n}$ and pulsation $\omega_{n}$ are linked by the dispersion relation:

$\beta_{n}^{4}=\frac{\omega_{n}^{2}}{\mu^{2}}$,

with $\mu=\sqrt{\frac{E I_{x}}{\rho A}}$ where $E$ is the Young's modulus of the cantilever material along its axis, $\rho$ its density, $I_{x}$ the moment of inertia of the cantilever in bending, and $A$ the cantilever's cross-section. The resonance frequencies associated to each mode are then:

$f_{n}=\frac{\omega_{n}}{2 \pi}$.

Fig. 2 shows the non-linear influence of the angle $\alpha$ on the 2 nd and 3rd resonance frequencies obtained from Eq. (1) for the parameters and

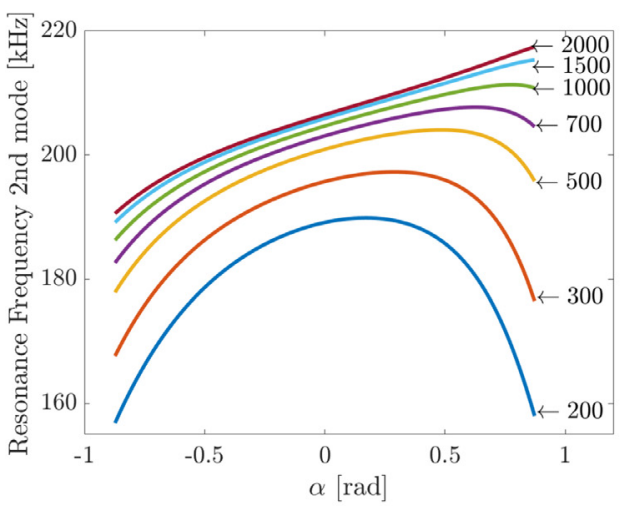

(a) range of ratio $k_{N} / k_{c}$ corresponding to our experimental conditions in the absence of friction or pinning $(\lambda=0)$. It is interesting to note how different the influence is for these two modes and how strongly it is dependent on the ratio $k_{N} / k_{c}$. The asymmetry related to the sign of the tilt angle arises from the asymmetry of the torque acting on the tip (projected normal force in the direction parallel to the cantilever times the height $h$ of the tip, $\sin \alpha$ term in the $C_{2}$ coefficient in Eq. (1) that cancels if $\lambda=1$, see Appendix A). The measure of the 3rd mode frequency enables us to study in good experimental condition the effect of the angle up to ratio $k_{N} / k_{c} \geq 2000$ (this would correspond to a contact modulus of about $50 \mathrm{GPa}$ in our experimental conditions).

\subsection{Tip-surface contact mechanics}

In this work, we modelled the tip-surface contact within the classical frame of elastic sphere-plane contact geometry. Assuming small amplitude excitation of less than a few nanometres, as it is the case in CR-AFM, and considering the rather stiff material with limited adhesion, contact was described by the DMT model [23]. The small modulation around the interaction force, which is the sum of the normal load $F_{N}$ and of the adhesive force $F_{a d h}$, allows then to express the normal stiffness as a function of this sum, of the tip radius $R_{t}$ and of the reduced modulus of the contact $E^{*}$, according to Johnson [24]:

$k_{N}=\sqrt[3]{6 R_{t}\left(F_{N}+F_{a d h}\right) E^{* 2}}$,

where the reduced modulus can be written as:

$E^{*}=\left(\frac{1}{M_{S}}+\frac{1}{M_{T}}\right)^{-1}$.

where $M_{S}$ and $M_{T}$ are the indentation moduli of the sample and tip, respectively. The indentation modulus of the silicon tip, oriented in (001) crystallographic direction, used in the model was $M_{T}=163 \mathrm{GPa}$.

For the investigation of the influence of the local slope, the interaction force has to be projected along the normal to the surface so that Eq. (4) is modified as:

$k_{N}=\sqrt[3]{6 R_{t}\left(F_{N}+F_{a d h}\right) \cos \phi E^{* 2}}$,

where $\phi$ is the local angle as described later in Fig. 4.

\section{Experimental measurements}

All measurements were performed with a SD-R30-CONT probe (Nano-sensors ${ }^{\mathrm{TM}}$ ). This type of probe has the advantage of having an initially rounded tip apex of a well-defined radius $R_{t}=(30 \pm 5) \mathrm{nm}$ obtained through manufacturing by ion beam melting. The cantilever is

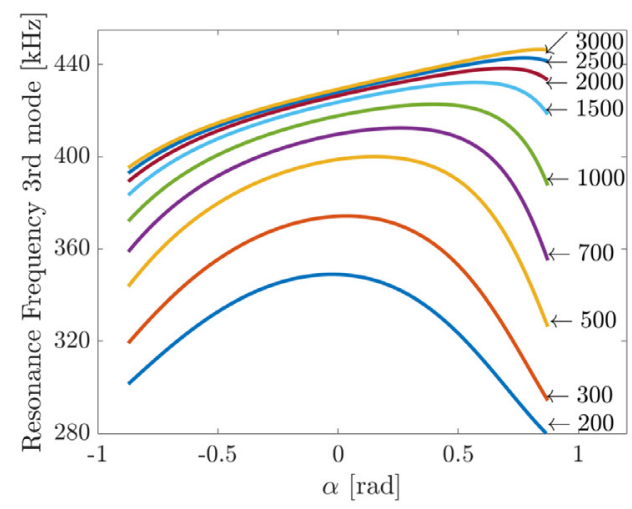

(b)

Fig. 2. Influence of the tip-surface angle on the (a) 2nd and (b) 3rd resonance frequency from Eq. (1) with $L_{1}=432 \mu \mathrm{m}$ and $h=12.5 \mu \mathrm{m}$ is shown for different ratios $k_{N} / k_{c}$ (values next to lines). Assuming a low cantilever stiffness of $k_{c}=0.27 \mathrm{Nm}^{-1}$ these ratios correspond to a range of indentation modulus $M_{S}$ of $1 \mathrm{GPa}-100 \mathrm{GPa}$. The second mode saturates for values of $k_{N} / k_{c}>1000$, the third mode for $k_{N} / k_{c}>2000$. 
rectangular, which is necessary to apply the developed model. Its spring constant was determined by thermal noise calibration [25] to be $k_{c}=0.27 \mathrm{~N} \mathrm{~m}^{-1}$, which is within the range of $0.02 \mathrm{~N} \mathrm{~m}^{-1}$ to $0.77 \mathrm{~N} \mathrm{~m}^{-1}$ given by the supplier. The cantilever length $L$ and tip height $h$ are supplied by the manufacturer as $L=(450 \pm 10) \mu \mathrm{m}$ and $h=(12.5 \pm 2.5) \mu \mathrm{m}$.

The resonance frequencies of the three first modes in free vibration were determined to be $f_{1}^{\text {free }}=13 \mathrm{kHz}$ (supplier: $6 \mathrm{kHz}-21 \mathrm{kHz}$ ), $f_{2}^{\text {free }}=86 \mathrm{kHz}$ and $f_{3}^{\text {free }}=243 \mathrm{kHz}$. These free vibration modes were used to determine experimentally the parameter $\mu=\sqrt{\frac{E I_{x}}{\rho A}}$ that is used in the calculation. We ensured that our values fitted very nicely the dispersion relation Eq. (2) relatively to the known theoretical wavelength of a cantilever of length $L$. We found $\mu=0.005 \mathrm{~m}^{2} \mathrm{~Hz}$, which is very close to the estimation $\left(\mu_{\text {est }}=0.0049 \mathrm{~m}^{2} \mathrm{~Hz}\right.$ ) that can be obtained from the cantilever characteristics.

CR-AFM was performed on a Bruker Dimension AFM with Nanoscope $\mathrm{V}$ controller with an averaged vertical force of $F_{N}+F_{a d h}=(200 \pm 10) \mathrm{nN}$. Sample holder discs containing the sample were placed on a small amount of acoustic coupling gel on top of a contact transducer (Olympus V 103-RM), which generated an ultrasonic sinusoidal oscillation. Resonance frequencies were tracked via dualresonance frequency tracking [26] using an H2FLI lock-in amplifier from Zürich instruments, which serves as a signal generator for the transducer excitation, too.

\subsection{Calibration of resonance frequencies}

The resonance frequencies of the tip-sample system are highly dependent on the exact geometry of the system. However, an accurate description of the contact mechanics of the tip surface system is difficult, if not unreachable, and analytical equations describing the whole range of geometry and mechanical properties are not available. In practice, most users avoid this problem by calibrating the resonance frequencies on surfaces with known mechanical properties for each cantilever $[13,27]$.

We performed the calibration of the cantilever by measuring resonance frequencies of the 2nd and 3rd modes of the cantilever on a number of rather flat surfaces at a constant scanning speed of $12 \mu \mathrm{m} \mathrm{s}^{-1}$. To obtain an accurate calibration the use of samples with not only a known indentation modulus, but also a surface with limited change of local angles and low roughness is important. The calibration samples were chosen so that their indentation moduli, determined by nano-indentation and/or using known elastic properties in the literature and anisotropic indentation model [28] if required, were in a range of 3 $\mathrm{GPa}-20 \mathrm{GPa}$. The samples and their respective indentation moduli are listed in Table 1.

The calibration points were then fitted with Eqs. (1) and (6) for the 2nd and 3rd modes simultaneously. Only the case where the lateral stiffness is null ( $\lambda=0$, free-sliding contact) is described here and represented in Fig. 3. The cases with $\lambda \neq 0$ are shown and discussed in the appendix (cf Appendix A), because it is shown in Section 3.2 that, with the present experimental conditions, only a free sliding contact describes the experimental data well. The best fit of the measured resonance frequencies versus the respective indentation modulus was

Table 1

Samples used for calibration of the resonance frequencies and their indentation moduli as determined by nano-indentation or from literature data.

\begin{tabular}{lllll}
\hline $\begin{array}{l}\text { Polystyrene } \\
\text { (PSFILM-12M, } \\
\text { Bruker) }\end{array}$ & $\begin{array}{l}\text { Acrylic resin (LR- } \\
\text { White, } \\
\text { Polysciences) [3] }\end{array}$ & $\begin{array}{l}\text { epoxy based } \\
\text { adhesive } \\
\text { (Hysol } \\
\text { EA9394) } \\
{[29]}\end{array}$ & PMMA [30] & $\begin{array}{l}\text { Mono- } \\
\text { crystalline } \\
\text { sulphur (100) } \\
{[30]}\end{array}$ \\
\hline $3 \mathrm{GPa}$ & $3.5 \mathrm{GPa}$ & $4.5 \mathrm{GPa}$ & $6.5 \mathrm{GPa}$ & $20 \mathrm{GPa}$ \\
\hline
\end{tabular}

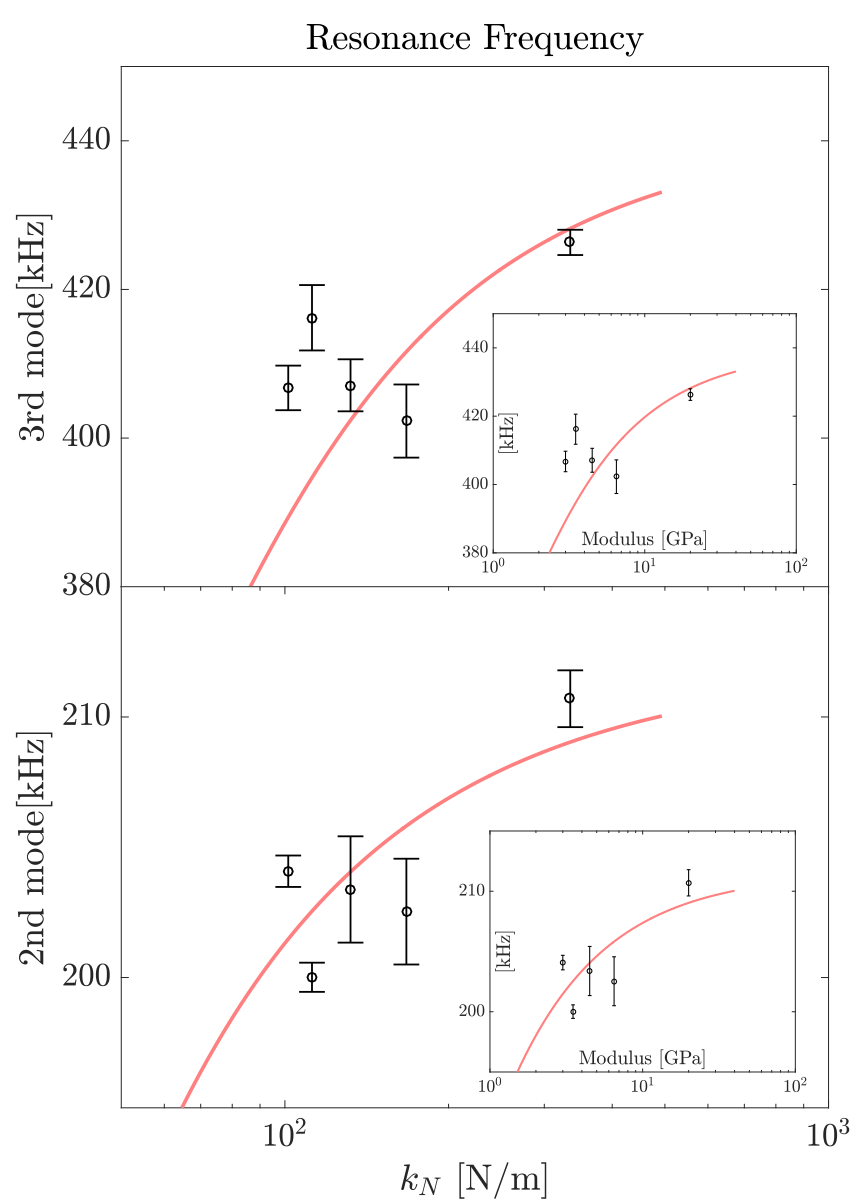

Fig. 3. Calibration curves for 2nd and 3rd CR-frequencies measured on samples with known indentation modulus. Black circles: experimental data; Red: fit with Eq. (1) to 2nd and 3rd mode simultaneously.

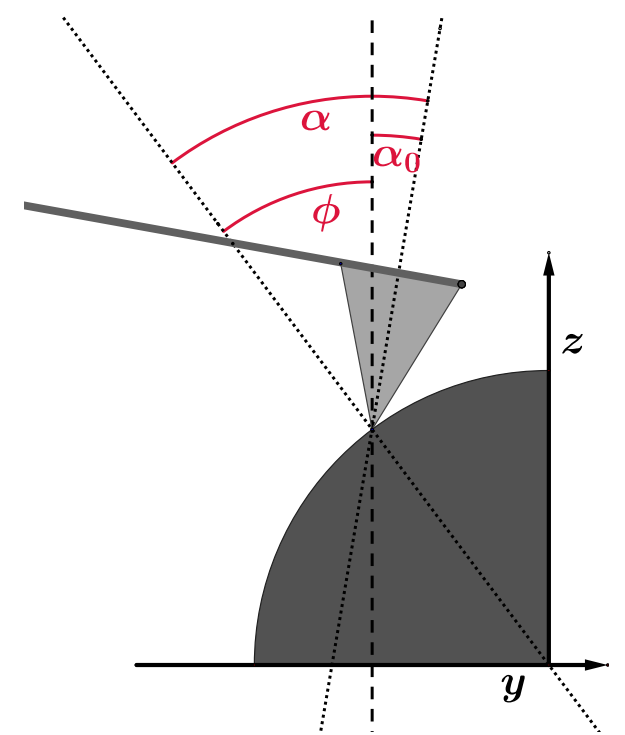

Fig. 4. Definition of angles in the direction parallel to the cantilever. $\alpha_{0}$ is resulting from the cantilever tilt in the AFM instrument and therefore constant. $\phi$ depicts the local angle. The resulting overall angle $\alpha$ is the sum of $\alpha_{0}$ and $\phi$.

obtained for the following parameters: $L_{1}=431 \mu \mathrm{m}, h=12.5 \mu \mathrm{m}$, $\alpha=9.7^{\circ}\left(:=\alpha_{0}\right)$ and $R_{t}=101 \mathrm{~nm}$.

The fitting values for $L_{1}$ and $h$ are in very good agreement with the supplier information on the cantilever and tip geometry, considering 
that the tip is generally located at a distance of $10 \mu \mathrm{m}$ to $20 \mu \mathrm{m}$ from the end of the cantilever. Considering that the total cantilever length is $L=(450 \pm 10) \mu \mathrm{m}$, this corresponds to a tip position parameter between 0.94 and 0.98 , in agreement with what is usually reported in the literature, e.g., 0.91-0.99 [31]. The inclination angle of the cantilever is known to be about $10^{\circ}$ in the Bruker Dimension AFM and is thus also in very good agreement with the fitting value found for the angle $\alpha$ on the horizontal samples. The radius found for the tip is three-times larger than the nominal tip radius specified by the supplier. This can be due to usual flattening of the far end of the tip apex that can be either the original state of the tip or caused by wear. Since tip geometry has been shown to play an important role in CR measurements [32], it was verified by an AFM measurement of the tip apex shape after CR measurements using a standard tip-check calibration grid (PA01, $\mu$ mash). We found that the far end of the tip (down to $0.5 \mathrm{~nm}$ depth corresponding to the maximal indentation depth on the different reference materials) was essentially spherical with a radius measured to be (92 \pm 15$) \mathrm{nm}$ which is in good agreement with the value $R_{t}=101 \mathrm{~nm}$ obtained by the fit, confirming the relevance of the chosen contact model in our measurement conditions.

One should notice that, even if the fit is not perfect compared to the experimental data, simultaneously fitting with the same set of parameters, which are sound when confronted to the known geometry of the tip/cantilever system, allows to describe well the level of the measured frequencies and the position of the curving point toward the plateau regime for the two modes. Noise in the resonance frequency determination was measured to be of the order of $1 \mathrm{kHz}$. Considering the calibration curves, we can thus infer that in our experimental conditions, frequency measurement will allow for determining contact moduli differences when these moduli are below $50 \mathrm{GPa}$ using the 3rd mode (respectively $30 \mathrm{GPa}$ using the 2 nd one). From the local slope of the curves, the sensitivity in terms of modulus determination can be evaluated to be around $0.1 \mathrm{MPa}$ at moduli of the order of $1 \mathrm{GPa}$ and of around $10 \mathrm{GPa}$ when we reach the limit of discrimination.

\subsection{Effect of local angle}

A curvature or slope of the sample surface changes the overall angle between tip and surface. As illustrated in Fig. 4, it must be considered as the sum of the inclination angle of the cantilever relative to the horizontal $\left(\alpha_{0}\right)$ and of the local angle of the surface towards the horizontal $(\phi)$. To study the effect of this local surface slope, CR-AFM was performed on spherical beads, which were assumed to be mechanically homogeneous. Hollow silica spheres with diameters of $10 \mu \mathrm{m}-30 \mu \mathrm{m}$ (Polysciences, Inc.) were selected for their high sphericity. They were spread onto double-sided sticky tape on a sample disc and sputtercoated with about $5 \mathrm{~nm}$ of gold for fixation of the beads. Topographical AFM scans were performed to select a bead with very good spherical appearance. Fig. 5 shows a three-dimensional view of the topography of the selected bead on the $12 \mu \mathrm{m} \times 12 \mu \mathrm{m}$ imaging area. The surface can be well fitted by a spherical cap of radius $r=13 \mu \mathrm{m}$, whose centre is very close to the centre of the image $\left(x_{0}=6.02 \mu \mathrm{m}, y_{0}=5.98 \mu \mathrm{m}\right)$. The root mean square difference between the fitted spherical cap and the real surface is $60 \mathrm{~nm}$.

The local angle $\phi$, defined in Fig. 4, can be obtained from any untreated topographical AFM image by calculating the partial derivative of the height $z$ with respect to the $y$-direction:

$\phi=\arctan \frac{\partial z}{\partial y}$

The angle measured by Eq. (7) on the selected bead can be compared to the theoretical value $\phi^{T h}$ calculated from the spherical fit according to:

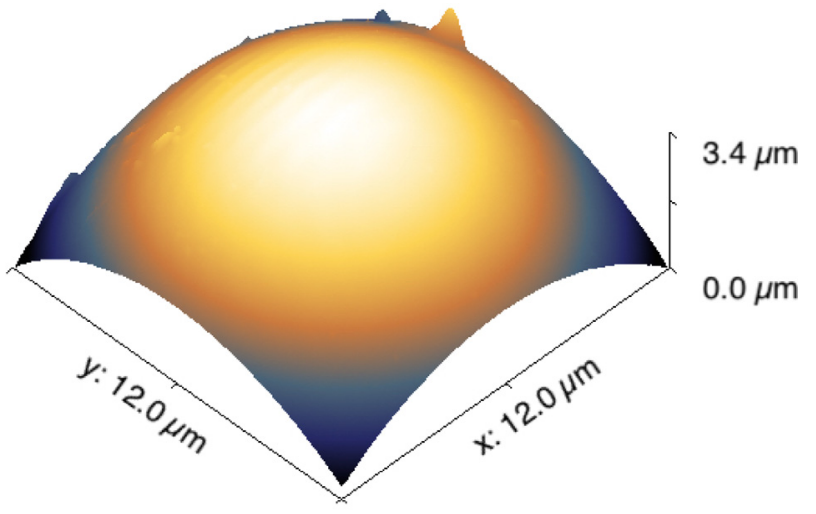

Fig. 5. 3D view of the topography of a silica glass bead measured by AFM. The surface can be well fitted to a sphere of radius $r=13 \mu \mathrm{m}$ centred at $x_{0}=6.02 \mu \mathrm{m}, y_{0}=5.98 \mu \mathrm{m}$.

$\phi^{T h}=-\arcsin \frac{y-y_{0}}{r \cos \left(\arcsin \frac{x-x_{0}}{r}\right)}$

The local angle determined by both methods is presented in Fig. 6 . The very good agreement between the two maps shows that the calculation of the partial derivative to obtain $\phi$ is reasonable.

Images of the 2nd and 3rd resonance frequency modes on the silica bead are presented in Fig. 7. In spite of the expected homogeneity of the surface in terms of mechanical properties, the measured frequencies are not constant and present a significant variation in the $y$-direction (along the cantilever axis, Fig. 4), whereas much fewer variations are observed in the $x$-direction (perpendicular to the cantilever axis, Fig. 4). This is the expected manifestation of the change of local angle relatively to the cantilever direction.

For each pixel of the image the 2nd and 3rd eigenmodes are measured independently, as well as the topography and therefore the local angle of the surface relatively to the cantilever. Such a set of images can thus be considered as an array of measurements of both frequencies as a function of the local angle on a material with homogeneous contact modulus $M_{S}$. Both frequency measurements are plotted as a function of the angle $\phi$ in Fig. 8. Due to the high number of pixels $(512 \times 512)$ and unavoidable scattering, iso-values of different point density are represented by a colour code. Regions of highest density are shown in yellow, lowest density in dark blue.

Both sets of data were fitted simultaneously with a least square optimization method, entering the values of $L_{1}, h, \alpha_{0}$ and $R_{t}$ that were determined during calibration (Section 3.1) as fixed parameters and using the sample contact moduli as the only fitting parameter for the model. Such fits were performed for increasing values of the lateral stiffness $(\lambda=0,0.3,0.5,1)$. The results are given in Appendix A. We observed that only in the case where the lateral stiffness is equal to zero, the fit was able to capture the main trend of the experimental data for both vibrational modes. This could be due to the fact that, in the present study, all the measurements and calibrations are done while scanning. It has been shown that ultrasonic vibrations lead to a reduction of friction even at the nanoscale [33-35]. Furthermore, the maximum modulus of reference materials used and measured is rather low (i.e., below $20 \mathrm{GPa}$ ) [13] and the ratio between the maximum contact stiffness and that of the cantilever is less than 10, i.e., before the plateau regime [36]. In those conditions, the contribution of the lateral stiffness and the pinning effect are assumed to be reduced. In consequence, the following part will be discussed in the frame of freesliding contact between the tip and the surface. The scattering of the data might be due not only to impurities at the surface, but also to geometrical effects such as the lateral angle and the variation of the contact area, which are not taken into account in the model. It should also be noted that the assumption of homogeneous mechanical response 


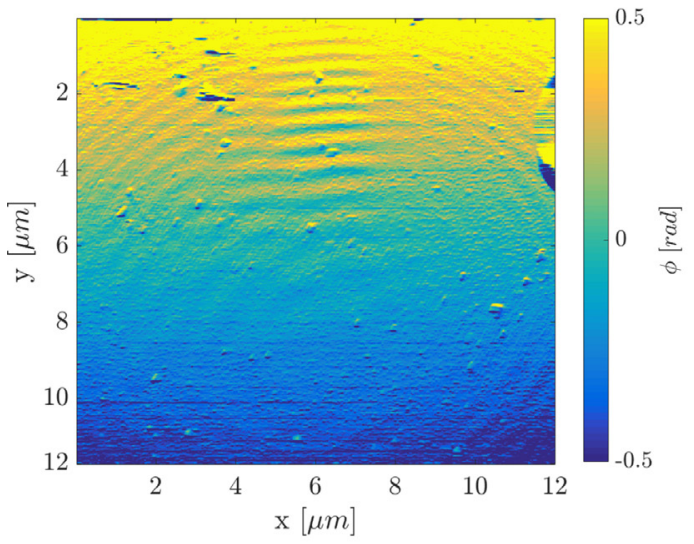

(a)

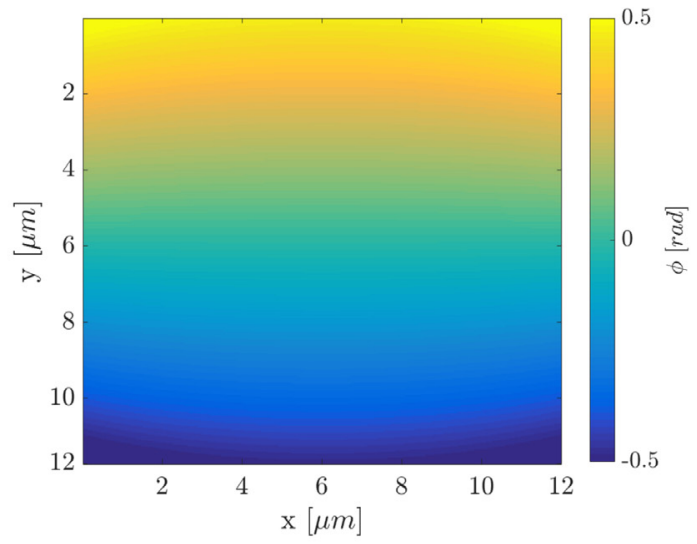

(b)

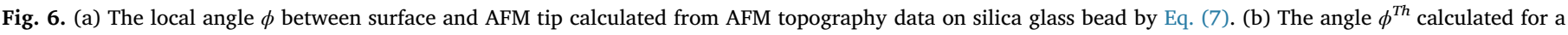
perfect sphere of the size of the measured silica glass sphere according to Eq. (8).

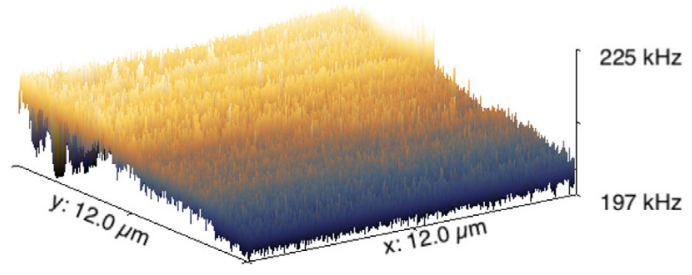

(a)

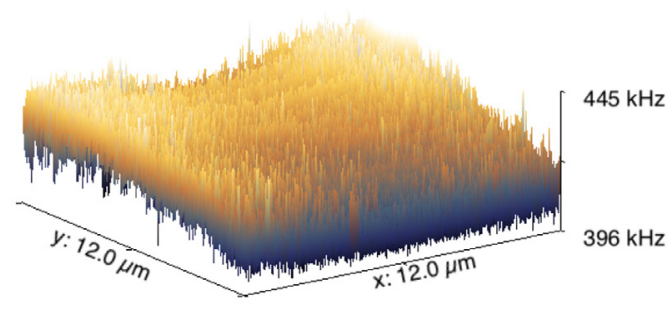

(b)

Fig. 7. Recorded CR-AFM frequencies for (a) 2nd and (b) 3rd mode on a silica glass bead.

of the sample over the scanning area is not accurate since we worked on an hollow sphere, as discussed hereafter. All these effects are likely to be the origin of the second order variation of the 3rd mode frequency observable along the $x$-axis in Fig. 7b. In any case, the model, even though it is simplified, captures the major trend of the data in both modes and can thus be used to correct the local slope artefact on the experimental measurements.

On the sphere, an apparent contact modulus of $M_{a}=(12 \pm 5) \mathrm{GPa}$ was found to provide the best simultaneous fit for the variation of the two frequency modes as a function of the local angle. This contact modulus, which corresponds to an apparent contact stiffness $k_{a}=(259 \pm 73) \mathrm{N} \mathrm{m}^{-1}$, does not correspond to the glass indentation modulus (i.e., $M_{\text {glass }}=73 \mathrm{GPa}$ ) as it results from the contact stiffness $k_{N}$ of the $5 \mathrm{~nm}$ gold layer in series with the stiffness $k_{S}$ of the hollow silica/ glass sphere, that behaves like a linear elastic membrane, with an effective mass $m_{S \text {, eff }}$ (Fig. 9) [37]. The sphere's exact shell thickness $h_{S}$ is unknown, but its order of magnitude was optically roughly estimated to be some hundreds of $\mathrm{nm}$. The theoretical stiffness $k_{S}$ of a thin hollow sphere, of radius $R_{S}$ and thickness $h_{S}$ loaded centrically, is available in the literature [38]:

$k_{S}=\frac{4 E_{\text {glass }} h_{S}^{2}}{\sqrt{3\left(1-v_{\text {glass }}^{2}\right)} R_{S}}$,

where $E_{\text {glass }}=71 \mathrm{GPa}$ and $\nu_{\text {glass }}=0.17$ for silica/glass elastic properties. In our case, quite high off-centre loading (up to $6 \mu \mathrm{m}$ ) occur during the scanning. Linear elastic finite elements modelling, using Cast3M 2017, ${ }^{1}$ of such a case yields an increase of $\approx 12 \%$ of the stiffness for an offcentred loading of $6 \mu \mathrm{m}$, which is therefore negligible here. In addition,

\footnotetext{
$\mathbf{1}$ http://www-cast3m.cea.fr/.
}

the applied load during CR-AFM measurements is such that there was only a slight bending of the hollow sphere relative to its radius. Its elastic response is thus linear. Moreover, eigen resonance frequencies of the pure glass hollow sphere can be estimated analytically [39]. This gives a resonance frequency of the first eigen mode around $47 \mathrm{MHz}$, i.e., the excitation frequencies of the AFM cantilever here are at least two order of magnitude lower. The behaviour of the hollow sphere can finally be assimilated to a linear spring without mass, i.e., in quasistatic. Thus, using Eq. (9), the indentation stiffness of the pure gold layer (Eq. (4) with $E_{\text {gold }}=77 \mathrm{GPa} \quad$ and $\quad \nu_{\text {gold }}=0.42, \quad M_{\text {gold }}=94 \mathrm{GPa}$, $F+F_{a d h}=200 \mathrm{nN}$ and $R_{t}=101 \mathrm{~nm}$ ) and the apparent contact stiffness, equivalent to the two springs $k_{N}$ and $k_{S}$ in series, of $259 \pm 73 \mathrm{~N} / \mathrm{m}$, it is possible to back calculate an estimate of the sphere shell thickness $h_{S}=(162 \pm 31) \mathrm{nm}$. This theoretical shell thickness agrees with the expected order of magnitude and validates the experimental measurements. One should note that the contribution of the gold layer and of the glass sphere are well decorrelated, as the indentation depth in the gold layer is estimated to be $\approx 0.3 \mathrm{~nm}$ in the worst case, which is at least ten times smaller than the gold layer thickness.

\subsection{Whole image correction}

From a complete set of data containing the frequencies (2nd and 3rd modes) and the local angle calculated from the topography via Eq. (7), a map of a corrected modulus can be calculated by solving Eq. (1) for each pixel separately. Fig. 10 compares the uncorrected estimation of the indentation modulus on the silica bead as obtained from both frequencies and constant cantilever tilt (Eq. (1) with $\alpha=\alpha_{0}$ ) to the corrected indentation modulus (Eq. (1) with $\alpha=\alpha_{0}+\phi$ for each pixel). Scatter excluded, the expected homogeneity of contact modulus on the scanned area is recovered after the correction. The average line profiles 


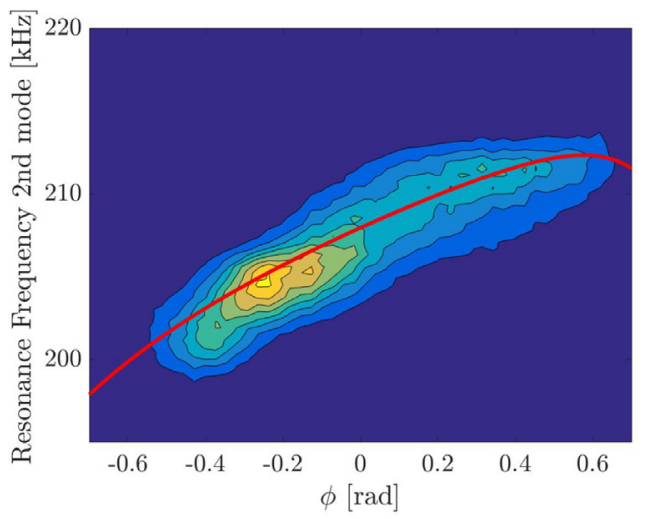

(a)

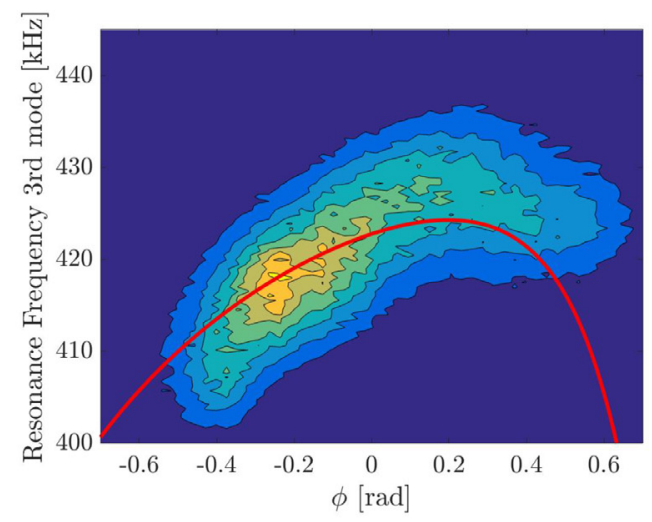

(b)
Fig. 8. Point density maps of the recorded CR-AFM frequencies plotted as a function of angle $\phi$ for (a) 2nd and (b) 3rd vibrational eigenmode on the silica glass bead. Densest areas are represented in yellow, least dense in dark blue. The result of a least square fit of Eq. (1) to the data is shown in red. (For interpretation of the references to colour in this figure legend, the reader is referred to the web version of this article.)

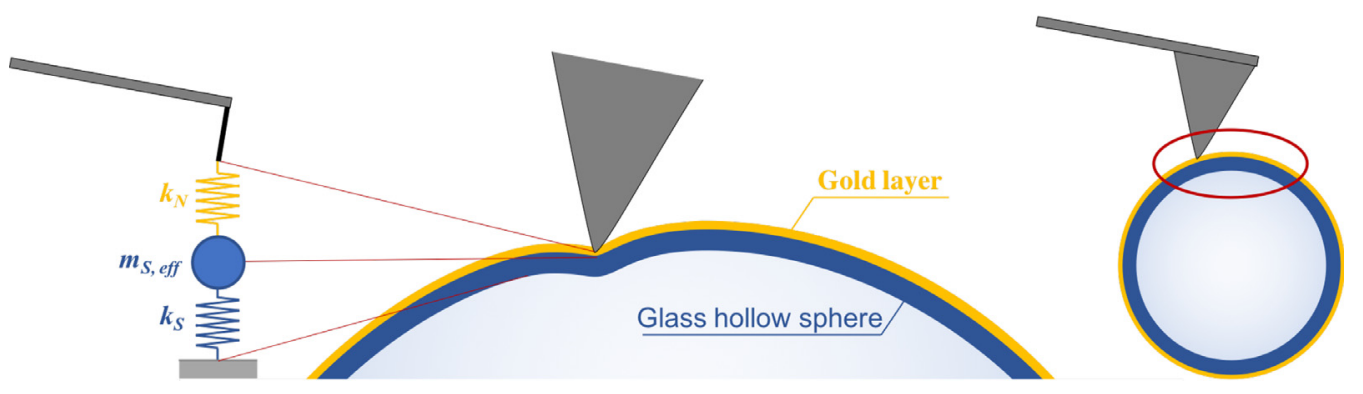

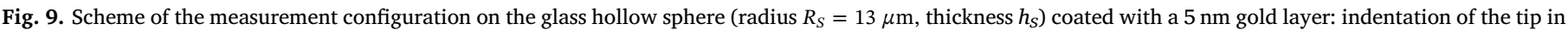
the gold layer, stiffness $k_{N}$, in series with the sphere elastic membrane, stiffness $k_{S}$ and effective mass $m_{S \text {, eff }}$, leading to an apparent contact stiffness $k_{a}$.

(Fig. 10c) show that the correction removes the inhomogeneities in the $y$-direction and that the corrected modulus value is more even throughout the whole image.

\section{Application}

The correction method was applied to the measurement of a biological surface, whose high surface slopes inhibit the interpretation of CR measurements so far: a cut starch granule within the sectioned endosperm of a durum wheat grain. Starch consists mainly of amylose and amylopectin, which together form spherical to elliptical granules in the grain [40]. Starch granules are, next to protein, one of the main components of wheat grains. Wheat grains are a natural composite material and the mechanical properties of the separate constituents are of interest in order to better understand the fragmentation behaviour during the milling process. Indentation modulus information on starch can provide insight into the elastic properties, which would complement other nano-mechanical measurements of for example the abrasive hardness of starch [41]. Nano-scale abrasion tests were able to reveal the overall differences in the abrasive hardness of starch $(2.0 \pm 0.6 \mathrm{GPa})$ and protein $(0.64 \pm 0.17 \mathrm{GPa})[42]$, but they are limited to measuring only the global properties on an area of $\sim 1 \times 1 \mu \mathrm{m}$. Both types of mechanical properties, elasticity and hardness, are necessary to accurately simulate the mechanical behaviour of the whole grain [7].

The sample was obtained by sectioning an intact wheat grain in a cryotome (Microm HM520, $-20^{\circ} \mathrm{C}$ ). Both distal regions of the grain (containing the brush and the germ [43]) were removed with a scalpel. The grain was then placed vertically on a cryotome sample holder, covered in freezing medium (Richard-Allan Scientific Neg-50TM) and placed in the cryotome for about $5 \mathrm{~min}$ to freeze fully. Sectioning was performed at slow speed using a carbon steel blade (Feather C35). The sectioning thickness was gradually reduced from $50 \mu \mathrm{m}$ to $2 \mu \mathrm{m}$ until a mirror-like surface was obtained.
As can be seen in Fig. 11 the starch granule exhibited high topography changes of several hundred nanometres within a small imaging area of $4 \mu \mathrm{m} \times 4 \mu \mathrm{m}$. Due to the centre of the granule being lower than the edges, both granule halves are strongly inclined at opposite angles and an additional overall inclination is present. Contact-resonance measurements on this sample are therefore greatly influenced by the surface slope and the correction is necessary to remove the artificial variation of the indentation modulus and to obtain the one of the actual material.

CR-AFM measurements were performed on the same set-up as described in Section 3 and with the same cantilever probe. The indentation moduli calculated without surface angle correction is shown in Fig. 12a. It shows a strong contrast between the left and right side of the granule, which is caused artificially by the opposite surface slopes of the granule halves. To obtain a meaningful modulus of the CR-AFM measurements on this area, the correction method for the whole image was applied on the data. The map of the modulus obtained after correction is shown in Fig. 12b and the histograms of the modulus before and after correction are shown in Fig. 12c. These figures show that our method allows for efficiently correct the asymmetry of the measured modulus induced by the different slopes of the opposing halves of the granule. The average value of indentation modulus obtained for this cut starch granule after correction was $(2.8 \pm 0.4) \mathrm{GPa}$. Within the granules itself, starch also exhibits nano-scale substructures, whose exact mechanical properties are still unknown. One of these substructures are growth rings, which originate from different states in the crystallinity of the starch polymers [40]. The rings' thickness is in the range of a few hundred nanometres and they are often already observable in topography images, the regions in-between two rings usually forming small valleys. It is possible that the regions of different crystallinity have different mechanical properties, but no direct evidence exists so far. One of the advantages of CR-AFM is the possibility to investigate the mechanical properties of such sub-micrometer structures. In the examined starch granule, growth rings were observed in the topography 


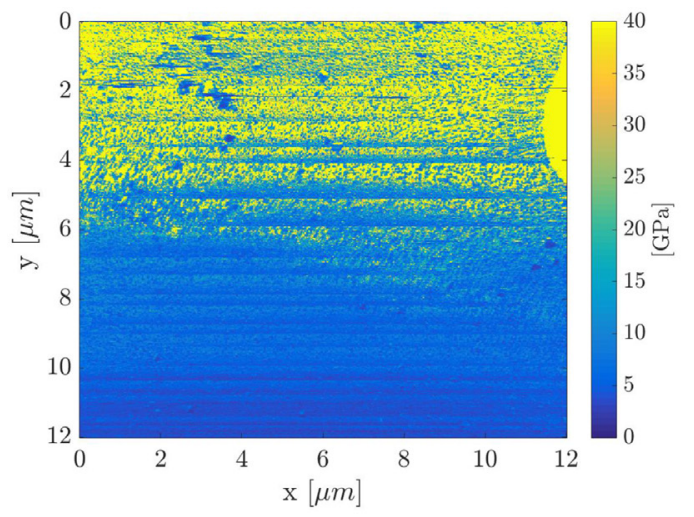

(a)

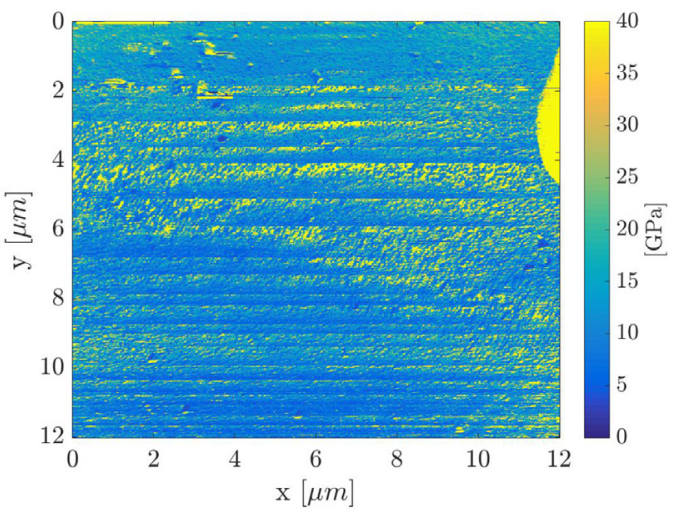

(b)

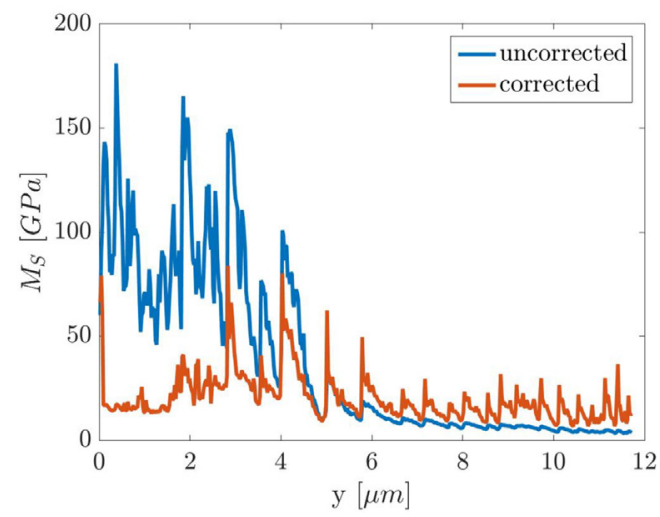

(c)

Fig. 10. (a) Uncorrected and (b) corrected maps of the indentation modulus on hollow silica bead are shown. The modulus is obtained by Eq. (1) for (a): $\alpha=$ const $=\alpha_{0}$ and (b): $\alpha=\alpha_{0}+\phi$, for each pixel respectively. The colour range is the same for both images. (c) shows the average line profile for both the uncorrected (blue line) and corrected (orange dashed line) modulus maps. (For interpretation of the references to colour in this figure legend, the reader is referred to the web version of this article.)

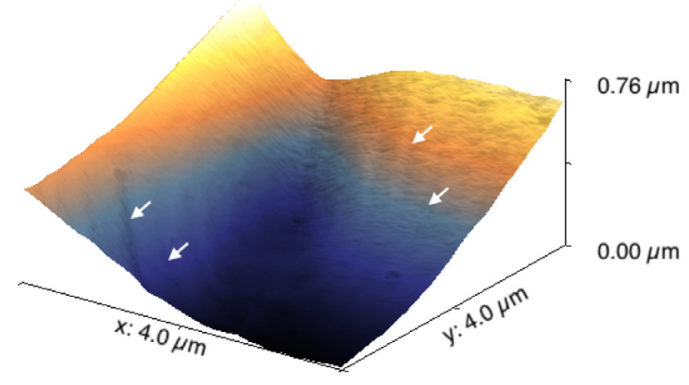

Fig. 11. Three-dimensional view of the topography on a cut starch granule in a sectioned wheat grain. Arrows are pointing out the valleys between growth rings.

image (Fig. 11). They were also recovered with contrast in the modulus maps without and, to a lesser extent, with surface slope correction (Fig. 12). The regions between the rings were slightly lower in modulus and the different crystallinity of the polymers could be the cause of these differences. However, the valleys between the rings are sharp ( $\sim 100 \mathrm{~nm}$ in width) and possibly lead to changes of the contact area, which are not corrected with our model.

The crystalline structure of starch and its deposition into circular growth rings result in an anisotropy of the material. Therefore, the measured mechanical properties are depending on the testing direction with regard to the orientation of the crystalline layers. This effect must also be taken into account in the interpretation of contact modulus measurements. Therefore, the real mechanical properties of the starch's growth rings cannot be determined yet by this method.

\section{Conclusion}

In this article we demonstrated the non-negligible effect of the surface angle, caused by high topography of the samples, on the mechanical measurements by CR-AFM. This effect is so pronounced that the obtained maps of the mechanical properties of curved surfaces become meaningless due to an artificial broadening of the moduli that can be measured, which has nothing to do with the actual mechanical properties of the sample. A simplified theoretical model, without damping, was shown to be able to predict the relation between surface angle and measured cantilever vibrational frequencies. The validity of the model was confirmed by comparison with experimental measurements on a spherical surface. Successful correction of the measurements on this surface demonstrated that the model is suitable to correct the mapping of the contact modulus on surface with local angles ranging from $-50^{\circ} \mathrm{C}$ to $50^{\circ} \mathrm{C}$ Furthermore, this model was successfully used to correct the experimental data on a surface of biological origin with high surface slopes. After correction, a first value of the indentation modulus of a starch granule in situ in a sectioned wheat grain was obtained. This opens the way to investigate possible differences in modulus of starch granules depending on the botanical origin.

We proposed thus a practical way to account for the local slope of the surface in the CR-AFM measurements. In our experimental conditions and for our sample, contact was likely to occur in free-sliding mode (i.e., with negligible lateral forces). We nevertheless show how 


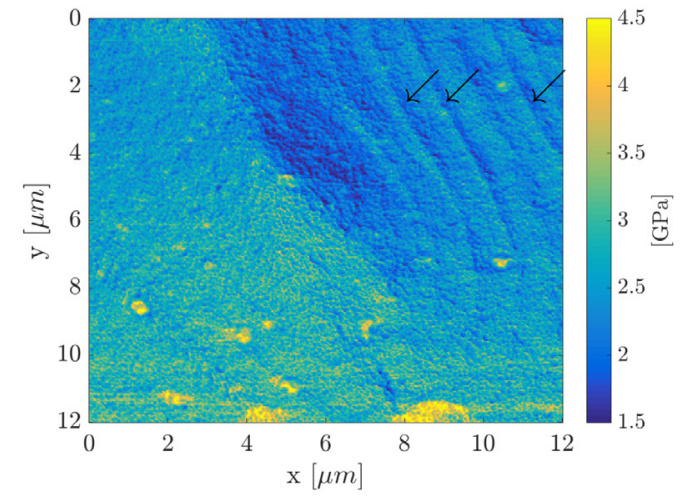

(a)

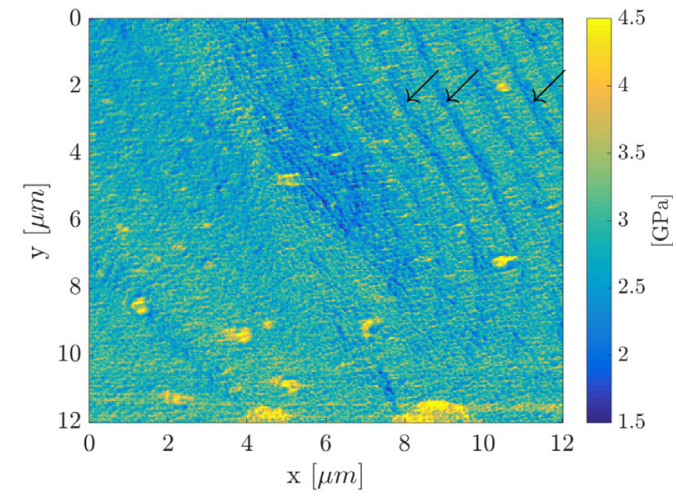

(b)

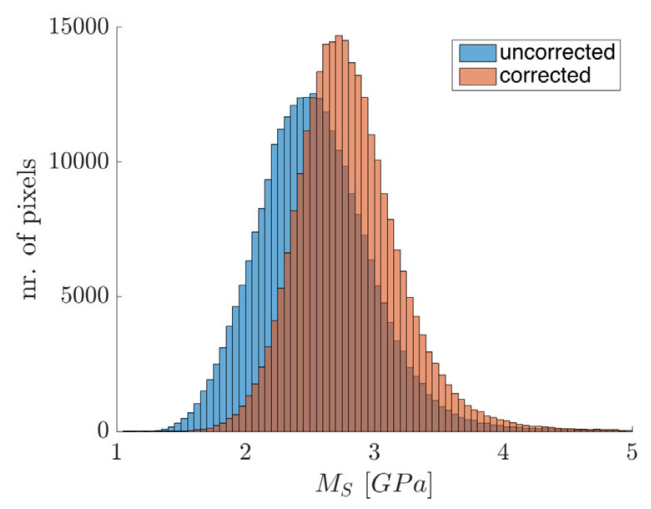

(c)

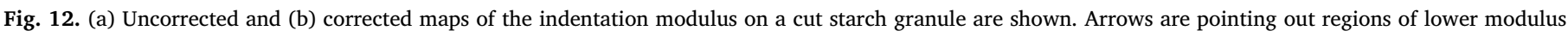
correlating to valleys between growth rings. (c) shows the modulus histograms of the uncorrected and corrected maps of the modulus.

these lateral forces can be accounted for and what is their influence on the relation between the local angle and the frequencies (cf Appendix A). It should be noticed that the proposed correction is likely to be effective only on topography with in plane typical length-scales of several hundreds of nanometres. Smaller curvatures caused by structures approaching the size of the tip diameter $(<100 \mathrm{~nm})$ might change the contact area in ways that are not corrected with this model [12].

The present study highlights in any case that extreme caution should be applied when interpreting nano-mechanical measurements by AFM, as the interactions between tip and sample on the nano-metre scale are highly complex and often non-linear. Similar effects are strongly expected to occur for other nano-mechanical AFM techniques, which rely on the effect of tip-sample contact on the cantilever behaviour, such as ultrasonic and acoustic AFM, PeakForce ${ }^{\mathrm{TM}} \mathrm{QNM}^{\mathrm{TM}}$ or $\mathrm{QI}^{\mathrm{TM}}, \mathrm{FMM}$ and other force-distance like modes.

\section{Acknowledgements}

We thank INRA (division for Science for Food and Bioproduct Engineering, grant $\mathrm{N}^{\circ} 140948$ ) and Labex Numev (grant $\mathrm{N}^{\circ}$ 2014-1-012) for the $\mathrm{PhD}$ grant for K. Heinze. We thank Richard Arinero (IES, Univ. Montpellier) for the fruitful exchange and the CIRAD Département BIOS/UMR AGAP/Plateforme PHIV (Montpellier, France) for cryotome sectioning.

\section{Appendix A. Effect of the lateral forces}

The whole analysis of the measured 2nd and 3rd modes resonance frequencies, from calibration to determination of the local angle effect on the spherical sample was performed for different values of the lateral/normal stiffness ratio $\lambda=0,0.3,0.5$ and 1 . Fits of calibration data for the two modes with increasing ratio $\lambda$ are presented in Fig. A.13. The accuracy of the calibration is not sufficient to allow for the discrimination between the different fits.

It is yet interesting to consider the fitting parameters which are calculated for each value of $\lambda$ and reported in Table A.2. It is in particular interesting to notice that for the intermediate cases, the fitting value of the tip radius, are very large, out of the range of the expected values.

For increasing value of the lateral/normal stiffness ratio $\lambda$ and consequent values of the geometrical parameters obtained from the calibration fit, we tried to adjust the experimental data obtained on the hollow sphere as a function of the local angle. The corresponding fitting curves are represented on Fig. A.14. We can remark that with the ratio $\lambda$ has a strong influence on the variation of the frequency as a function of the local angle. It was expected, since this ratio change drastically the torque applied on the cantilever/tip system. Moreover, the stronger the lateral forces are set in the model, the less the fit is able to capture the global trend of the data. In our experimental conditions this leads us to think that the tip/surface contact while scanning was in free sliding mode. It would nevertheless be interesting to systematically investigate the influence of the lateral force when correcting the data, especially if adhesive surfaces are tested. 


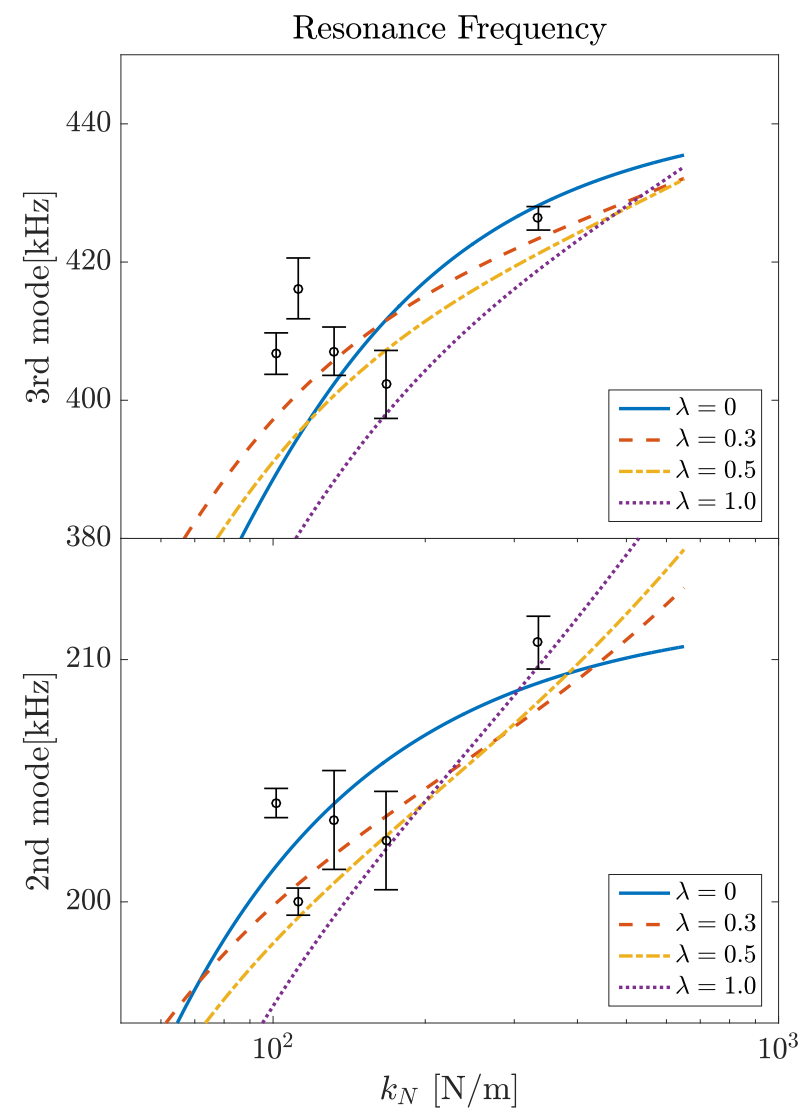

Fig. A1. Calibration curves for 2nd and 3rd CR-frequencies measured on samples with known modulus for values of $\lambda$ between 0 and 1 is shown. Black circles show the experimental data with error bars.

Table A1

Fitting parameter of the calibration data as a function of stiffness ratio $\lambda$.

\begin{tabular}{lllll}
\hline$\lambda$ & 0 & 0.3 & 0.5 & 1 \\
\hline$R_{t}(\mathrm{~nm})$ & 101 & 403 & 279 & 76 \\
$L_{1}(\mu \mathrm{m})$ & 431 & 438 & 438 & 436 \\
$\alpha_{0}\left({ }^{\circ}\right)$ & 9.7 & 8.6 & 6.9 & 14.2 \\
\hline
\end{tabular}

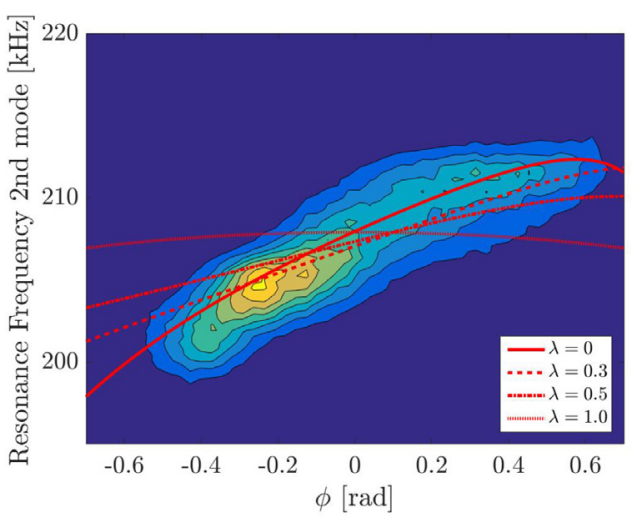

(a)

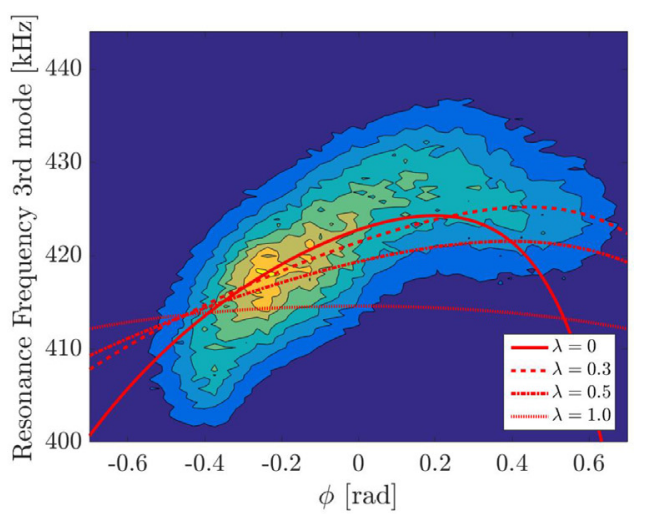

(b)
Fig. A2. Point density maps of the recorded CR-AFM frequencies on the silica glass bead plotted as a function of angle $\alpha$ for (a) 2nd and (b) 3rd vibrational mode. Results of least square fits for values of $\lambda$ between 0 and 1 are shown in red. (For interpretation of the references to colour in this figure legend, the reader is referred to the web version of this article.)

\section{References}

[1] K. Radotić, C. Roduit, J. Simonović, P. Hornitschek, C. Fankhauser, D. Mutavdžić, G. Steinbach, G. Dietler, S. Kasas, Atomic force microscopy stiffness tomography on living Arabidopsis thaliana cells reveals the mechanical properties of surface and deep cell-wall layers during growth, Biophys. J. 103 (3) (2012) 386-394.

[2] P. Milani, M. Gholamirad, J. Traas, A. Arnéodo, A. Boudaoud, F. Argoul, O. Hamant, In vivo analysis of local wall stiffness at the shoot apical meristem in arabidopsis using atomic force microscopy, Plant J. 67 (6) (2011) 1116-1123, https://doi.org/ 10.1111/j.1365-313X.2011.04649.x.

[3] O. Arnould, R. Arinero, Towards a better understanding of wood cell wall 
characterisation with contact resonance atomic force microscopy, Compos. Part A 74 (2015) 69-76.

[4] S.S. Nair, S. Wang, D.C. Hurley, Nanoscale characterization of natural fibers and their composites using contact-resonance force microscopy, Compos. Part A 41 (5) (2010) 624-631.

[5] W. Rodríguez-Castellanos, F.J. Flores-Ruiz, F. Martínez-Bustos, F. Chiñas-Castillo, F.J. Espinoza-Beltrán, Nanomechanical properties and thermal stability of recycled cellulose reinforced starch-gelatin polymer composite, J. Appl. Polym. Sci. 132 (14) (2015).

[6] O. Arnould, D. Siniscalco, A. Bourmaud, A.L. Duigou, C. Baley, Better insight into the nano-mechanical properties of flax fibre cell walls, Ind. Crops Prod. 97 (2017) 224-228, https://doi.org/10.1016/j.indcrop.2016.12.020.

[7] J. Delenne, Y. Haddad, J. Bénet, J. Abecassis, Use of mechanics of cohesive granular media for analysis of hardness and vitreousness of wheat endosperm, J. Cereal Sci. 47 (3) (2008) 438-444.

[8] U. Rabe, Atomic Force Acoustic Microscopy, Springer Berlin Heidelberg, Berlin, Heidelberg, pp. 37-90.

[9] D.C. Hurley, Contact Resonance Force Microscopy Techniques for Nanomechanical Measurements, Springer Berlin Heidelberg, Berlin, Heidelberg, pp. 97-138.

[10] J.A. Turner, J.S. Wiehn, Sensitivity of flexural and torsional vibration modes of atomic force microscope cantilevers to surface stiffness variations, Nanotechnology 12 (3) (2001) 322.

[11] P.-E. Mazeran, L. Odoni, J.-L. Loubet, Curvature radius analysis for scanning probe microscopy, Surf. Sci. 585 (1-2) (2005) 25-37.

[12] G. Stan, R.F. Cook, Mapping the elastic properties of granular Au films by contact resonance atomic force microscopy, Nanotechnology 19 (23) (2008) 235701.

[13] D. Passeri, M. Rossi, J. Vlassak, On the tip calibration for accurate modulus measurement by contact resonance atomic force microscopy, Ultramicroscopy 128 (2013) 32-41.

[14] B.D. Huey, AFM and acoustics: fast, quantitative nanomechanical mapping, Annu. Rev. Mater. Sci. 37 (2007) 351-385.

[15] K. Yamanaka, A. Noguchi, T. Tsuji, T. Koike, T. Goto, Quantitative material characterization by ultrasonic AFM, Surf. Interface Anal. 27 (5-6) (1999) 600-606, https://doi.org/10.1002/(SICI)1096-9918(199905/06)27:5/6<600::AIDSIA508 > 3.0.CO; $2-W$.

[16] U. Rabe, S. Amelio, E. Kester, V. Scherer, S. Hirsekorn, W. Arnold, Quantitative determination of contact stiffness using atomic force acoustic microscopy, Ultrasonics 38 (1-8) (2000) 430-437.

[17] P.A. Yuya, D.C. Hurley, J.A. Turner, Contact-resonance atomic force microscopy for viscoelasticity, J. Appl. Phys. 104 (7) (2008) 074916.

[18] J.P. Killgore, D.G. Yablon, A.H. Tsou, A. Gannepalli, P.A. Yuya, J.A. Turner, R. Proksch, D.C. Hurley, Viscoelastic property mapping with contact resonance force microscopy, Langmuir 27 (23) (2011) 13983-13987.

[19] F. Dinelli, M.R. Castell, D.A. Ritchie, N.J. Mason, G.A.D. Briggs, O.V. Kolosov, Mapping surface elastic properties of stiff and compliant materials on the nanoscale using ultrasonic force microscopy, Philos. Mag. A 80 (10) (2000) 2299-2323, https://doi.org/10.1080/01418610008216474.

[20] T.J. Young, M.A. Monclus, T.L. Burnett, W.R. Broughton, S.L. Ogin, P.A. Smith, The use of the PeakForce ${ }^{\mathrm{TM}}$ quantitative nanomechanical mapping AFM-based method for high-resolution Young's modulus measurement of polymers, Meas. Sci. Technol. 22 (12) (2011) 125703.

[21] G. Smolyakov, C. Formosa-Dague, C. Severac, R. Duval, E. Dague, High speed indentation measures by FV, QI and QNM introduce a new understanding of bionanomechanical experiments, Micron 85 (Supplement C) (2016) 8-14, https://doi. org/10.1016/j.micron.2016.03.002.
[22] M. Radmacher, R. Tillmann, H. Gaub, Imaging viscoelasticity by force modulation with the atomic force microscope, Biophys. J. 64 (3) (1993) 735-742.

[23] E. Barthel, Adhesive elastic contacts: JKR and more, J. Phys. D 41 (16) (2008) 163001.

[24] K.L. Johnson, Contact Mechanics, Cambridge University Press, 1985.

[25] J.E. Sader, J.W.M. Chon, P. Mulvaney, Calibration of rectangular atomic force microscope cantilevers, Rev. Sci. Instrum. 70 (10) (1999) 3967-3969.

[26] B.J. Rodriguez, C. Callahan, S.V. Kalinin, R. Proksch, Dual-frequency resonancetracking atomic force microscopy, Nanotechnology 18 (47) (2007) 475504.

[27] D.C. Hurley, Quantitative Measurements of Elastic Properties with Ultrasonic-Based AFM and Conventional Techniques, Springer Berlin Heidelberg, Berlin, Heidelberg, pp. 351-373.

[28] A. Delafargue, F.-J. Ulm, Explicit approximations of the indentation modulus of elastically orthotropic solids for conical indenters, Int. J. Solids Struct. 41 (26) (2004) 7351-7360, https://doi.org/10.1016/j.ijsolstr.2004.06.019.

[29] J. Jumel, G. Bresson, O. Arnould, W. Bou Mjahed, N. Ben Salem, M.E. Shanahan, Hysol EA9394 strength under multiaxial proportional loading statistical analysis in relation with microstructural analysis, 5th International Symposium on Advanced Science and Technology in Experimental Mechanics, (2010).

[30] R. Arinero, G. Leveque, Vibration of the cantilever in force modulation microscopy analysis by a finite element model, Rev. Sci. Instrum. 74 (1) (2003) 104-111, https://doi.org/10.1063/1.1525874.

[31] D.C. Hurley, K. Shen, N.M. Jennett, J.A. Turner, Atomic force acoustic microscopy methods to determine thin-film elastic properties, J. Appl. Phys. 94 (4) (2003) 2347-2354, https://doi.org/10.1063/1.1592632.

[32] M. Kopycinska-Muller, R.H. Geiss, D.C. Hurley, Contact mechanics and tip shape in AFM-based nanomechanical measurements, Ultramicroscopy 106 (2006) 466-474.

[33] F. Dinelli, S.K. Biswas, G.A.D. Briggs, O.V. Kolosov, Ultrasound induced lubricity in microscopic contact, Appl. Phys. Lett. 71 (9) (1997) 1177-1179, https://doi.org/ 10.1063/1.120417.

[34] A. Socoliuc, E. Gnecco, S. Maier, O. Pfeiffer, A. Baratoff, R. Bennewitz, E. Meyer, Atomic-scale control of friction by actuation of nanometer-sized contacts, Science 313 (5784) (2006) 207-210, https://doi.org/10.1126/science.1125874.

[35] M.T. Cuberes, Nanoscale Friction and Ultrasonics, Springer International Publishing, pp. 35-55. doi:10.1007/978-3-319-10560-4_3.

[36] J.P. Killgore, D.C. Hurley, Low-force AFM nanomechanics with higher-eigenmode contact resonance spectroscopy, Nanotechnology 23 (5) (2012) 10. 055702

[37] A. Striegler, B. Koehler, B. Bendjus, M. Roellig, M. Kopycinska-Mueller, N. Meyendorf, Detection of buried reference structures by use of atomic force acoustic microscopy, Ultramicroscopy 111 (8) (2011) 1405-1416, https://doi.org/ 10.1016/j.ultramic.2011.05.009.

[38] L. Zhang, M. D’Acunzi, M. Kappl, G.K. Auernhammer, D. Vollmer, C.M. van Kats, A. van Blaaderen, Hollow silica spheres: synthesis and mechanical properties, Langmuir 25 (5) (2009) 2711-2717.

[39] S. Hayek, Vibrations of a spherical shell in acoustic medium, J. Acoust. Soc. Am. 40 (2) (1966) 342-348.

[40] A. Buléon, P. Colonna, V. Planchot, S. Ball, Starch granules: structure and biosynthesis, Int. J. Biol. Macromol. 23 (2) (1998) 85-112.

[41] E. Chichti, M. George, J.-Y. Delenne, V. Lullien-Pellerin, Changes in the starchprotein interface depending on common wheat grain hardness revealed using atomic force microscopy, Plant Sci. 239 (2015) 1-8.

[42] E. Chichti, M. George, J.-Y. Delenne, F. Radjai, V. Lullien-Pellerin, Nano-mechanical properties of starch and gluten biopolymers from atomic force microscopy, Eur. Polym. J. 49 (12) (2013) 3788-3795.

[43] T. Swift, M.U.D. of Botany, Histology of the Wheat Grain, Monash University, 1969. 